\title{
Characterisation of adhesion receptors mediating lymphocyte adhesion to bronchial endothelium provides evidence for a distinct lung homing pathway
}

\author{
M P Ainslie, C A McNulty, T Huynh, F A Symon, A J Wardlaw
}

Thorax 2002;57:1054-1059

See end of article for authors' affiliations

Correspondence to: Correspondence to:
Professor A Wardlaw Department of Respiratory Medicine, Glenfield Hospital, Groby Road, Leicester LE3 9QP, UK; aw24@le.ac.uk

Revised version received 1 July 2002

Accepted for publication

31 July 2002

\begin{abstract}
Background: The lung is an important tertiary lymphoid organ and many lung diseases are associated with disordered lung immunity. Unlike the gut ( $\alpha 4 \beta 7$ binding to MAdCAM-1) and skin (CLA+ve T cells binding to E-selectin) where the adhesion receptors involved in organ specific homing of $T$ cells have been identified, the molecular pathways controlling lymphocyte migration to the lung are unclear. Using a modified version of the Stamper-Woodruff assay we have investigated the receptors mediating adhesion of peripheral blood lymphocytes to airway endothelium.

Methods: Longitudinal frozen sections of bronchus $(8 \mu \mathrm{m})$ obtained from lung resection specimens were incubated with $\mathrm{T}$ cell enriched peripheral blood mononuclear cells for 30 minutes under shaking conditions in the presence of a fluorescently labelled polyclonal anti-von Willebrand antibody to identify blood vessels. After fixation the percentage of blood vessels supporting adhesion was measured. Blocking monoclonal antibodies were used to determine the role of individual adhesion receptors in lymphocyte binding.

Results: Specific cation dependent binding of lymphocytes to bronchial endothelium was observed which was significantly inhibited by antibodies against P-selectin, PSGL-1, L-selectin, LFA-1, ICAM-1 and ICAM-2 but not E-selectin, VLA-4, VCAM-1 or Mac-1. This was consistent with the pattern of endothelial expression of these receptors with strong expression of P-selectin and ICAM-1, but negligible expression of E-selectin on bronchial endothelium.

Conclusion: This study suggests an important role for PSGL-1/P-selectin in T cell migration into the bronchi and provides further evidence for a pattern of recirculation for respiratory tract homing $T$ cells distinct from the gut and skin.
\end{abstract}

$\Lambda^{1}$ though the lung is primarily an organ of gas exchange, it also has an important secondary function as a route of sensitisation for airborne antigens and pathogens. Consistent with this role, lung tissue contains large numbers of memory lymphocytes and lung lymphatics drain into abundant groups of perihilar lymph nodes. ${ }^{1}$ The lung is therefore a major "tertiary" lymphoid organ. Perhaps as a result, many lung diseases including asthma, sarcoidosis, and tuberculosis are associated with disordered $\mathrm{T}$ cell immunity. ${ }^{2} \mathrm{~T}$ lymphocytes continually recirculate, trafficking between the blood and tissue compartments. ${ }^{3}$ Memory and naïve T lymphocytes have distinct circulatory patterns. Whereas naïve cells are restricted to entering secondary lymphoid tissues via specialised high endothelial vessels, a subset of CCR7 negative memory cells can also enter non-specialised lymphoid tissues. ${ }^{4}$ Tissue homing memory cells have been shown to preferentially migrate back to the site of their initial antigenic stimulation, a process called lymphocyte homing, which teleologically increases the chance of a memory cell reencountering the antigen to which it was sensitised. Lymphocyte homing is controlled by the adhesion receptors and chemokines associated with the multistep process involved in leucocyte extravasation. ${ }^{5}$ The first step involves tethering of the leucocyte to the endothelium which, in the case of $\mathrm{T}$ cells, is mediated primarily by the selectins with Pand E-selectin expressed on the endothelium and L-selectin on the leucocyte. ${ }^{6}$ Their ligands are mucin-like glycoproteins which are decorated with a family of O-glycosylated carbohydrate moieties related to sialyl Lewis $X^{7}$ The selectin ligands include P-selectin glycoprotein 1 (PSGL-1) which binds all three selectins but has the greatest affinity for P-selectin. ${ }^{89}$ Alpha-4 integrins can also mediate the tethering $\operatorname{step}^{10} ; \alpha 4 \beta 1$ binds vascular cell adhesion molecule 1 (VCAM-1) which is widely expressed on inflamed endothelium, ${ }^{11}$ and $\alpha 4 \beta 7$ binds mucosal addressin cell adhesion molecule l (MAdCAM-1) which is selectively expressed on gut endothelium. ${ }^{12}$ Following the tethering step, $\mathrm{T}$ cells become activated by endothelially expressed chemokines. ${ }^{13}{ }^{14}$ This allows engagement of lymphocyte function antigen (LFA-1) binding to intercellular adhesion molecule (ICAM)-1 and ICAM-2 which leads to firm arrest and transmigration under the direction of a tissue expressed chemokine. The molecular signals that control lymphocyte homing to lymph nodes, the gut, and skin are becoming increasingly well defined. ${ }^{315}$ Thus homing of naïve cells to peripheral lymph nodes is selectively controlled by L-selectin binding to a number of mucin-like ligands selectively expressed on high endothelial vessels, ${ }^{16}{ }^{17}$ and CCL21 binding to CCR7 which is expressed by all naïve cells and a proportion of memory cells. ${ }^{13} 18$ The gut homing pathway involves $\alpha 4 \beta 7 /$ MAdCAM-1 and CCL25/CCR9. ${ }^{19} 20$ Skin homing T cells are defined by expression of cutaneous lymphocyte antigen (CLA) which is expressed on a glycoform of PSGL-1 and binds E-selectin which is preferentially expressed on skin endothelium. ${ }^{21}$ CCR4 binding to CCL17 mediates the arrest

Abbreviations: CLA, cutaneous lymphocyte antigen; EGTA, ethylene glycol aminoethyltetraacetic acid; FSA, frozen section assay; ICAM, intercellular adhesion molecule; LFA-1, lymphocyte function antigen 1; Mac-1, macrophage antigen 1; MAdCAM-1, mucosal addressin cell adhesion molecule 1; PECAM-1, platelet endothelial cell adhesion molecule; PSGL-1, P-selectin glycoprotein 1; VCAM-1, vascular cell adhesion molecule 1 ; VLA-4, very late antigen 4 . 
step of skin homing T cells and CCR10 binding to CCL27 the chemotaxis step. ${ }^{22} 23$

Although used extensively in studies of lung inflammation, the mouse has not been a good model of homeostatic lymphocyte homing to the lung because of non-specific trapping in pulmonary capillaries and because, unlike human lung, the normal mouse bronchial lamina propria is a flimsy structure which contains relatively few lymphocytes. However, studies in other animal models have suggested a distinct pattern of re-circulation for respiratory tract associated T cells. For example, in rat nasal associated lymphoid tissue (NALT), T cell binding was L-selectin but not MAdCAM- 1 dependent, suggesting closer links to peripheral than to gut lymph nodes. ${ }^{24}$ In the sheep, whereas gut associated T cells were $\alpha 4 \beta 7$ high, L-selectin low and T cells from peripheral lymph nodes were $\alpha 4 \beta 7$ low, L-selectin high, T cells from lung lymph were low for both receptors, suggesting a distinct migration profile. ${ }^{25}$ No specific lung lymphocyte homing receptors have been described, but lung $\mathrm{T}$ cells do have a distinctive adhesion and CR phenotype suggesting the existence of a migration pathway distinct from the gut and skin. Thus, they express low levels of $\alpha 4 \beta 7$ and L-selectin and are negative for CLA. ${ }^{26}$ We have found that lung resection and bronchoalveolar lavage (BAL) derived T cells express low to moderate levels of CCR4 and are generally negative for CCR9 and CCR7. They therefore have a chemokine receptor profile which differs from gut, skin, and lymph node homing $\mathrm{T}$ cells. ${ }^{27}$ This suggests that, while there appears to be a specific population of lung homing lymphocytes, a lung specific homing receptor remains to be identified.

To investigate the adhesion receptors involved in homing of $\mathrm{T}$ cells to the airway, we examined adhesion of peripheral blood lymphocytes to bronchial endothelium using a modified version of the frozen section Stamper-Woodruff assay (FSA) that has been used by many groups including ours to investigate leucocyte adhesion in a physiological setting. ${ }^{28}$ We have found that the tethering step is mediated primarily by PSGL-1 binding to P-selectin with a contribution from L-selectin, and that the arrest step is mediated by LFA-1 binding to ICAM-1 and ICAM-2.

\section{METHODS}

\section{Tissue}

Large and medium sized bronchus was obtained from patients undergoing lung resections for cancer performed at Glenfield Hospital, Leicester, UK. No other clinical details were available. Samples were macroscopically and microscopically free of tumour infiltration. These were cut into small $\mathrm{l} \mathrm{cm}^{2}$ sections and snap frozen in liquid nitrogen with a longitudinal orientation. On the day of the experiment sections were cut as required using a Bright cryostat. The study was approved by the Leicestershire Health ethics committee.

\section{Reagents}

FSA antibodies: WAPS (anti-P-selectin), E-134 (anti-Eselectin), and Dreg 56 (anti-L-selectin) were gifts from Eugene Butcher; 4B9 (VCAM-1) was a gift from Roy Lobb Biogen; PLl (PSGL-1), HP2/1 (anti-VLA-4), 7E4 (anti-CD18), 84H10 (ICAM-1), BT-1 (ICAM-2) were purchased from Immunotech; MOPC 21 from Sigma; 2LPM19C (Mac-1) and G25.2 (LFA-1) from Dako. All these antibodies were IgG isotype and were used at a final concentration of $10 \mu \mathrm{g} / \mathrm{ml}$ which was optimal for all the antibodies used.

Antibodies used in panning: TuK4, DJ130c, HD37 and MOC-1 all from Dako.

VWF-FITC was obtained from Biodesign; Vector shield fluorescent mountant (Vector Labs); Histopaque 1077 from Sigma; glutaraldehyde from Fisher; biotinylated rabbit anti-mouse ABC solution from Dako.
In vitro frozen section assay of lymphocyte endothelial binding

The endothelial binding assay was a modified version of that previously described..$^{29}$ Briefly, peripheral blood lymphocytes were suspended at $7 \times 10^{6}$ cells $/ \mathrm{ml}$ in medium 199 (M199) and $2 \%$ fetal calf serum (FCS). They were incubated for 30 minutes at room temperature on freshly cut unfixed $8 \mu \mathrm{m}$ frozen sections of bronchus under rotating conditions using a "Belly Dancer" shaker at $60 \mathrm{rpm}$. An ethylene glycol aminoethyltetraacetic acid (EGTA) control was included to assess the level of non-specific binding. To allow blood vessels to be identified a FITC conjugated polyclonal antibody against von Willebrand factor was co-incubated with the lymphocytes to allow identification of blood vessels (in preliminary experiments a phycoerythrin (PE) conjugated antibody was used). In experiments involving antibody blockade, those directed against lymphocytes were incubated with the cells for 30 minutes on ice before addition to sections and endothelial directed antibodies were incubated for 30 minutes with the sections on ice before the T cells were added. After 30 minutes non-adherent cells were detached by gently tipping the fluid off the sections followed by fixation in $2 \%$ glutaraldehyde in phosphate buffered saline. No counterstain was used. Lymphocyte binding was expressed as the percentage of blood vessels with two or more cells bound.

Cell binding to endothelium was determined by first identifying a blood vessel using fluorescent microscopy and then switching to light microscopy where adherent lymphocytes could be readily identified lying just above the plane of the tissue. Cells were only scored as binding if they were in direct contact with the endothelium. 100 blood vessels were counted per section. All conditions were used on triplicate sections with one section per slide and the mean was taken as a single data point. All slides were counted blind by a single observer. The coefficient of variation for the assay was $13.3 \%$. It was not possible to do more than about 18 slides per experiment because the slides would become unevenly shaken and the plates on which they were held would be unstable on the shaker. The maximum number of conditions per experiment was therefore six including the EGTA and IgG control.

\section{Histopaque separation of human peripheral blood mononuclear cells}

Blood (50-100 ml) was obtained from normal human volunteers using standard venesection techniques. The collected blood was layered over 1.077 density Histopaque (Sigma). After centrifugation at $400 \mathrm{~g}$ at room temperature for $30 \mathrm{~min}$ utes, leucocytes were collected from the buffy layer and washed twice with M199/2\% FCS.

\section{Purification of lymphocytes}

A number of approaches were taken to enrich $\mathrm{T}$ cells in the mononuclear cell fraction. Our standard conditions involved depletion of monocytes by two rounds of adherence to tissue culture plastic at $37^{\circ} \mathrm{C}, 5 \% \mathrm{CO}_{2}$ for 30 minutes. In some experiments human $\mathrm{T}$ cell enrichment columns (R\&D Systems) were used to obtain a $\mathrm{T}$ cell pure fraction according to the manufacturers' instructions. Panning was also used to obtain a $\mathrm{T}$ cell enriched population. Briefly, petri dishes were coated with $8 \mathrm{ml}$ rabbit anti-mouse antibody, buffered at pH $9(80 \mu \mathrm{l}$ antibody diluted in $8 \mathrm{ml}$ bicarbonate solution). The dishes were left overnight at $4^{\circ} \mathrm{C}$ to allow the antibody to adhere fully to the plastic. The excess antibody was washed from the surface and the plate was blocked with $8 \mathrm{ml} \mathrm{M199/2 \%} \mathrm{FCS} \mathrm{for} 3$ hours at $4^{\circ} \mathrm{C}$, after which the dish was washed twice. Monocyte depleted mononuclear cells at $1 \times 10^{8}$ cells $/ \mathrm{ml}$ were then incubated with antibodies against human CD14, CD19, and $\mathrm{CD} 56$ at $10 \mu \mathrm{g} / \mathrm{ml}$ for 45 minutes at $4^{\circ} \mathrm{C}$. The cells were layered on the petri dish (capacity $3 \times 10^{7}$ cells) and incubated at $4^{\circ} \mathrm{C}$ for 1 hour. The non-adherent $\mathrm{T}$ cell enriched population was obtained by gentle washing. 
Table 1 Expression of adhesion receptors on bronchial lamina propria endothelium

\begin{tabular}{llll}
\hline $\begin{array}{l}\text { Adhesion } \\
\text { receptor }\end{array}$ & $\begin{array}{l}\text { No of tissues } \\
\text { stained }\end{array}$ & $\begin{array}{l}\text { Antibody } \\
\text { clone }\end{array}$ & $\begin{array}{l}\text { Intensity of } \\
\text { expression (median)* }\end{array}$ \\
\hline IgG control & 9 & MOPC21 & 1 \\
PECAM-1 & 9 & EN4 & 5 \\
ICAM-1 & 8 & 84 HIO & 3 \\
ICAM-2 & 6 & BT-1 & 2.5 \\
VCAM-1 & 8 & $4 B 9$ & 2.5 \\
P-selectin & 9 & WAPS & 4 \\
E-selectin & 9 & E-134 & 2 \\
\hline
\end{tabular}

PECAM = platelet endothelial cell adhesion molecule,

ICAM=intercellular adhesion molecule; VCAM=vascular cell adhesion molecule.

* $0=$ no staining; 1 =just detectable; 2 =clearly detectable but weak

$3=$ good staining; $4=$ strong staining; $5=$ very strong staining.

Monocyte depletion resulted in $75-80 \%$ purity of $\mathrm{T}$ cells as determined by flow cytometry. R\&D columns resulted in up to $90 \%$ purity of $\mathrm{T}$ cells, but yields were low with a recovery of approximately $30 \%$. A combination of panning and monocyte depletion resulted in good recovery and a purity of up to $88 \%$. Monocyte depletion was the method used unless stated in the text or figure legends (data not shown).

\section{Immunostaining of bronchus}

Frozen sections of lung tissue $(6 \mu \mathrm{m})$ were fixed in acetone (Analar Grade, Merck) for 10 minutes and washed in Tris-buffered saline (TBS) before blocking with $100 \mu \mathrm{l}$ rabbit serum (1:100) for 10 minutes at room temperature. After blocking, excess serum was removed and $100 \mu \mathrm{l}$ TBS diluted antibody was incubated with the sections for 60 minutes under gentle rocking. Excess unbound antibody was removed with two washes of TBS and $100 \mu \mathrm{l}$ biotinylated $\mathrm{F}\left(\mathrm{ab}^{\prime}\right)_{2}$ rabbit anti-mouse antibody (1:200) was incubated for 30 minutes. The staining was amplified using standard $\mathrm{ABC}$ technique and a new fuschin substrate. The sections were counterstained with haematoxylin and examined under a light microscope at $\times 20$ magnification. The intensity of expression on the blood vessels was measured using a subjective assessment as previously described ${ }^{30}$ with $0=$ no staining, $1=$ just detectable, $2=$ clearly detectable but weak, $3=$ good staining, $4=$ strong staining, and $5=$ very strong staining. A mouse control antibody was used as a reference for negative staining and EN4 ( an antibody which recognises PECAM-1 and strongly stains all blood vessels) as a positive control.

\section{Statistical analysis}

Lymphocyte binding under various conditions was expressed as the mean (SE) value of at least three separate experiments undertaken with different tissue sources and blood donors. Statistical significance was ascertained using a two tailed Student's $t$ test and a p value of $<0.05$ was regarded as significant.

\section{RESULTS}

\section{Immunostaining}

To determine which adhesion receptors were expressed by airway endothelium, we immunostained bronchial tissue with antibodies against endothelial adhesion receptors. P-selectin, E-selectin, and ICAM-2 expression was confined to blood vessels in the lamina propria whereas ICAM-1 was also expressed on leucocytes and epithelium. VCAM-1 was widely expressed on endothelium, smooth muscle, and bronchial epithelium. P-selectin was consistently highly expressed whereas E-selectin expression was either weak or no greater than the mouse Ig control. VCAM-1 and ICAM-2 expression were weak to moderate whereas ICAM-1 was well expressed (table 1).

\section{Specificity of binding of T cells to bronchial endothelium}

T lymphocytes could be readily identified binding to blood vessels. This binding was inhibited by EGTA, indicating that it was cation dependent and therefore likely to be mediated by adhesion receptor interactions (figs 1 and 2). Binding of cells in the absence of a blocking antibody or chelation of cations was in the region of $40-50 \%$ (mean 45.1 (SE 4.3 )\%). This was reduced to a background level of 10-19\% (mean 14 (SE 4)\%) by EGTA.
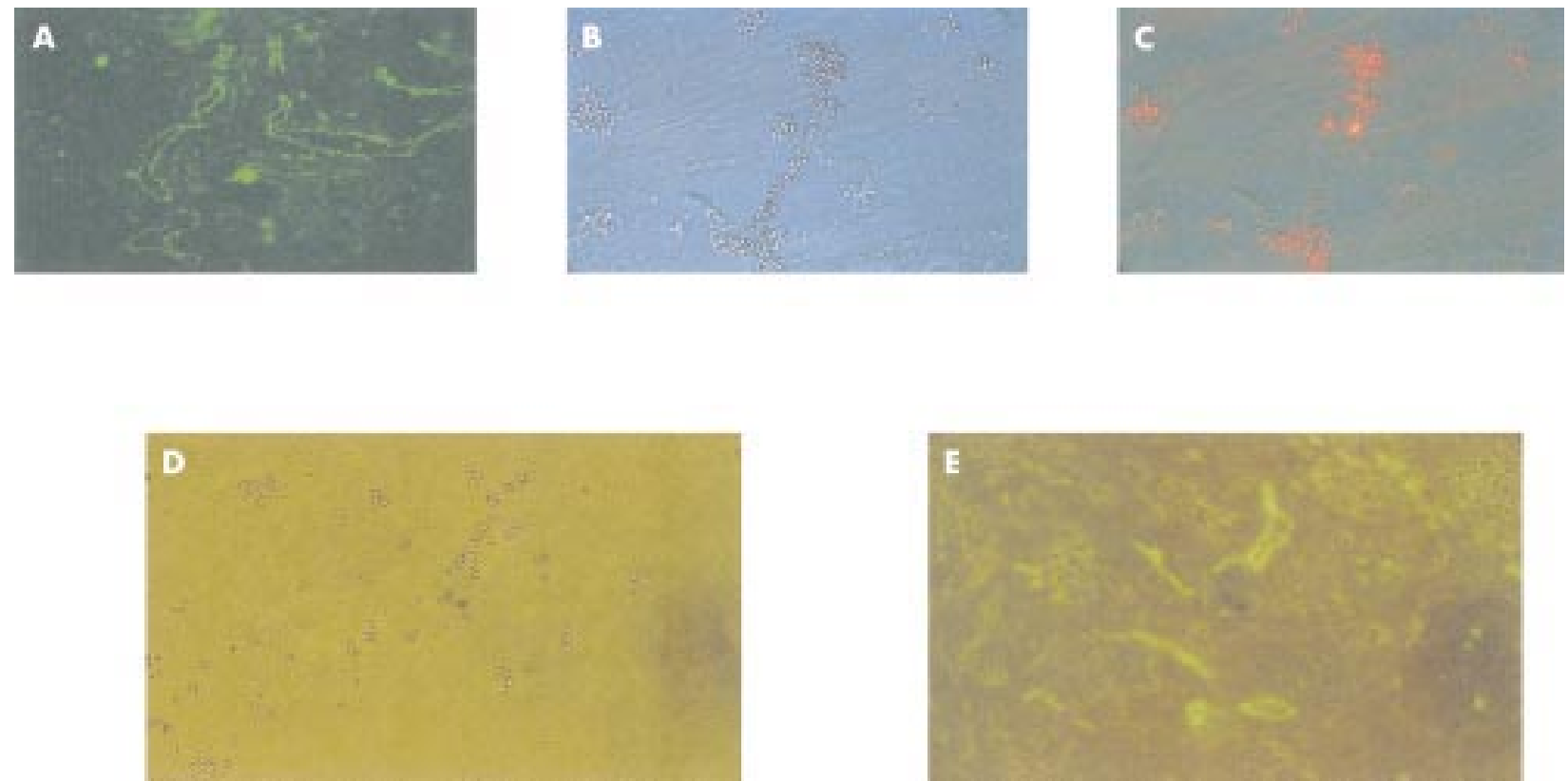

Figure 1 Photographs of lymphocytes binding to bronchial endothelium. (A) Staining of an 8 um section of a bronchus cut longitudinally with a polyclonal antibody against von Willebrand factor directly conjugated to FITC showing the rich plexus of blood vessels in the bronchial submucosa. (B) and (C), (D) and (E) Two paired examples of lymphocytes binding to adjacent sections of bronchus with one of each pair (C and E) treated with EGTA to inhibit adhesion receptor mediated binding. In one of the experiments (B/C) the anti-von Willebrand antibody was conjugated to PE. The photographs show leucocytes binding specifically to the blood vessels stained by the antibody but not in the adjacent areas. Cell binding is inhibited by EGTA. 


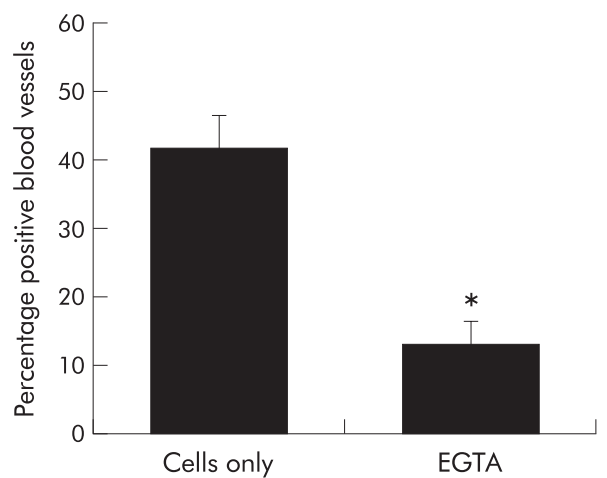

Figure 2 Inhibition of lymphocyte binding to airway endothelium by EGTA. Monocyte depleted peripheral blood lymphocytes were added to sections of bronchus and the percentage of blood vessels supporting adhesion were counted in triplicate slides and the mean calculated. The results are expressed as the mean (SE) of four experiments. ${ }^{*} p<0.05$.

\section{Identification of the adhesion receptors mediating $\mathrm{T}$ cell tethering}

To identify the adhesion receptors mediating the adhesion of $\mathrm{T}$ cells to bronchial endothelium we first used monoclonal antibodies against the receptors involved in leucocyte tethering. Antibodies against PSGL-1, the major ligand for P-selectin, and L-selectin both caused about $50 \%$ inhibition of specific binding compared with the EGTA control, whereas an antibody against VLA-4 had no effect (fig 3). When antibodies against PSGL-1 and L-selectin were used together, no additive effect was observed (IgG control 50\% binding; EGTA 19\%; PSGL-1 32\%; L-selectin 37\%; PSGL-1 + L-selectin 33\%; mean of $n=2$ ).

Experiments using cells purified with the R\&D columns gave similar results with inhibition with both anti-PSGL-1 and anti-L-selectin monoclonal antibodies (data not shown).

Consistent with the blocking effect of anti-PSGL-1 and L-selectin, antibodies against P-selectin consistently and significantly blocked adhesion with a mean inhibition of binding of $72 \%$ relative to the EGTA control. In contrast, antibodies against VCAM-1 and E-selectin were ineffective (fig 4).

\section{Characterising the adhesion receptors involved in} arrest of $T$ cells on lung endothelium

We have previously noted that the FSA can model both the tethering and arrest steps of the adhesion paradigm. We

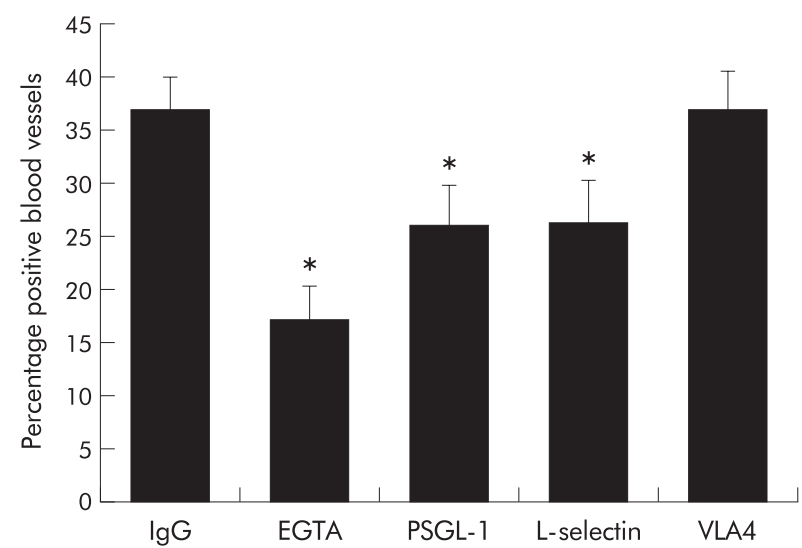

Figure 3 Mediation of lymphocyte adhesion by PSGL-1 and L-selectin. Monocyte depleted peripheral blood lymphocytes were incubated with blocking antibodies for 30 minutes before adding to the section of bronchus. Experiments were done in triplicate and expressed as mean (SE) of three experiments. lgG in this as in subsequent figures refers to the $\lg _{1}$ isotype control mouse $\lg G$ monoclonal antibody. ${ }^{*} p<0.05$.

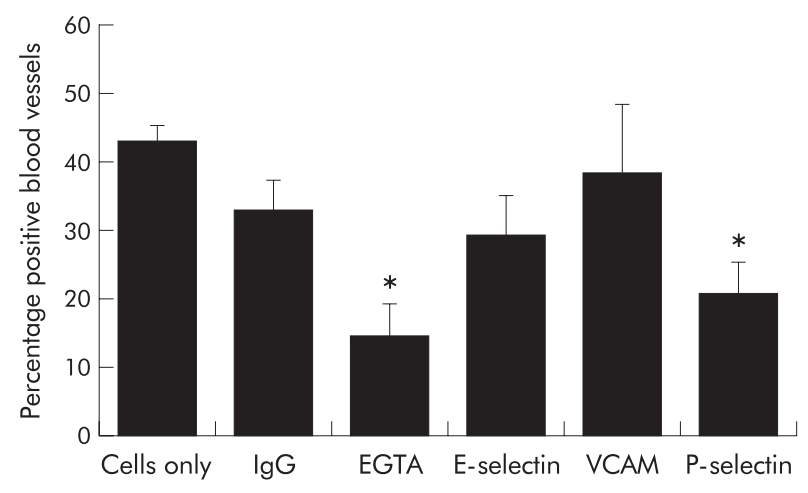

Figure 4 Mediation of lymphocyte adhesion by P-selectin. Monocyte depleted peripheral blood lymphocytes were added to frozen sections of bronchus which had been incubated for 30 minutes with blocking antibodies against the indicated receptors. Experiments were done on triplicate sections and the results are expressed as the mean (SE) of three experiments. ${ }^{*} p<0.05$.

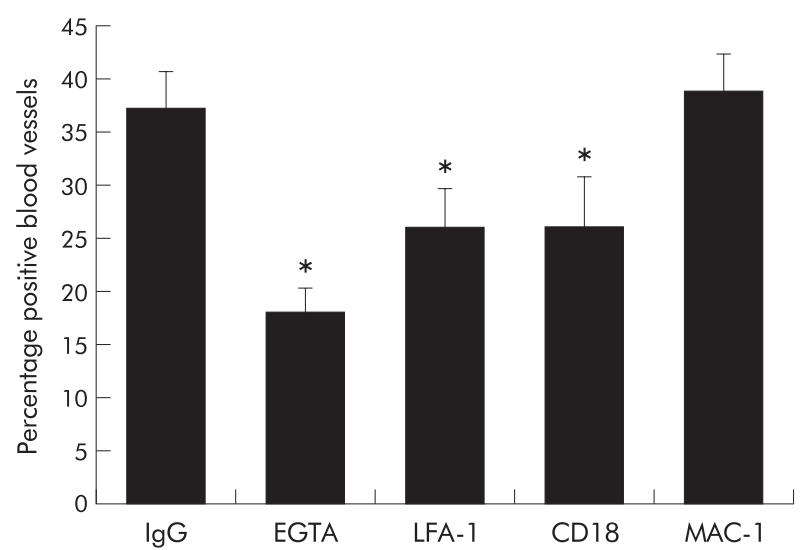

Figure 5 Mediation of lymphocyte adhesion by LFA-1. Peripheral blood lymphocytes were enriched for T cells by monocyte depletion followed by panning. They were then incubated with blocking monoclonal antibodies against the $\alpha$ chain of LFA-1, the $\alpha$ chain of Mac-1, or the common CD18 $\beta$ chain. Each condition was done on triplicate slides and the results expressed as the mean (SE) of four experiments. ${ }^{*} p<0.05$.

therefore examined whether LFA- 1 and its endothelial ligands ICAM- 1 and ICAM-2 were involved in T cell binding to airway endothelium. Blocking monoclonal antibodies against LFA-1 and CD18 but not Mac-1 significantly inhibited T cell adhesion (fig 5). Adhesion using the monoclonal antibody against LFA-1 and CDI8 was very similar. In addition, antibodies against ICAM-1 and ICAM-2 both significantly inhibited adhesion (fig 6).

\section{DISCUSSION}

The molecular signals controlling T cell trafficking through the lung both during normal homeostatic homing and inflammation are still unclear. In this study we report the development of an ex vivo assay, based on the FSA developed by Stamper and Woodruff, which we have used to determine the adhesion receptors involved in $\mathrm{T}$ cell adhesion to bronchial endothelium in humans in a physiological setting. Using this novel approach we have made the original observation that PSGL-1 and L-selectin interacting with P-selectin are potential tethering receptors, and LFA- 1 binding to both ICAM- 1 and ICAM-2 are likely to be involved in cell arrest.

We have previously used the FSA to investigate the adhesion and activation signals involved in leucocyte adhesion to nasal polyp endothelium. ${ }^{29}{ }^{31}$ Adapting this assay to the lung 


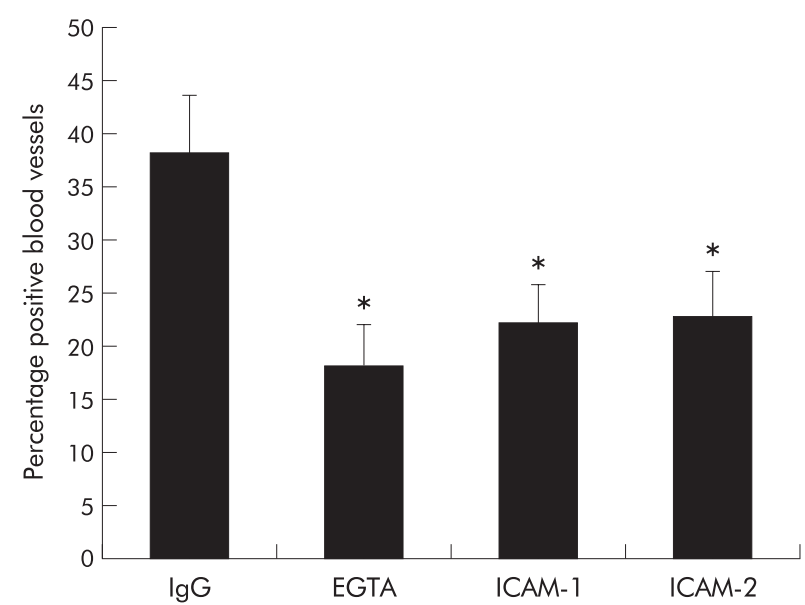

Figure 6 Mediation of lymphocyte adhesion by ICAM-1 and ICAM-2. Monocyte depleted peripheral blood lymphocytes were added to $8 \mu \mathrm{m}$ sections of bronchus treated for 30 minutes with a blocking antibody against either ICAM-1 or ICAM-2. Experiments were undertaken using triplicate slides and results were expressed as mean (SE) of four experiments. ${ }^{*} p<0.05$.

was more difficult, however, firstly because of the presence of additional structures such as smooth muscle, cartilage and mucus glands which are not present in the nasal polyp, and secondly because, unlike the nasal mucosa, blood vessels could not be identified in the bronchial lamina propria using a simple counterstain such as MayGrunewald/Giemsa. In order to solve this problem we used a fluorescent labelled polyclonal antibody against a pan endothelial marker (von Willebrand factor) which stained blood vessels extremely brightly and was therefore able to overcome the background immunofluorescence in the section. This allowed precise colocalisation of leucocytes and blood vessels by the expedient of switching rapidly between light and fluorescent microscopy and altering focus to detect cells just above the plane of the tissue. This resulted in a reproducible assay with an acceptable coefficient of variation, although the tendency of cells to clump in the vicinity of the structural components in the airway is the likely explanation for a higher background (adhesion remaining after EGTA) than in the nasal polyp assay, and we cannot exclude the possibility that $\mathrm{T}$ cells were adhering through an alternative non-cation dependent mechanism. Manipulation of the peripheral blood lymphocytes appeared to increase this background and, for this reason, most of our experiments involved the use of an enriched $\mathrm{T}$ cell population obtained by monocyte depletion. This technique resulted in $75-80 \%$ purity, the remaining cells being largely B cells and NK cells. However, in experiments where we used methods which gave higher purities, similar results were obtained so we are confident that we are measuring predominantly $\mathrm{T}$ cell adhesion related events. Nevertheless, as we were not using a $100 \%$ pure $\mathrm{T}$ cell population, we cannot rule out the possibility that the adherent cells were contaminating B cells or NK cells. We have attempted on a number of occasions in both lung and nasal polyps to stain adherent cells in situ using immunofluorescent markers, but we have been unable to do this because of an unacceptably high background fluorescence which meant there was a similar signal from the cells stained with the IgG control as with the anti CD3 antibody.

Our findings are very similar to those we have previously reported for $\mathrm{T}$ cell binding to the nasal mucosa with an important role for L-selectin, P-selectin, and PSGL-1 as well as LFA-1, but a minor or non-existent role for VLA-4/VCAM-1 and E-selectin. ${ }^{29}$ This is consistent with the pattern of expression of these receptors. We found that P-selectin was consistently highly expressed throughout the airway, whereas VCAM-1 expression was variable and very little expression of
E-selectin was observed. This raises the possibility that $\mathrm{P}$-selectin is the tethering receptor in the airway in the same way that E-selectin is important in this role in the skin. PSGL- 1 is the major receptor for P-selectin, however the ligand being recognised by L-selectin is less clear as all human L-selectin endothelial ligands characterised so far are restricted in their expression to lymph node high endothelial vessels. ${ }^{16}$ PSGL- 1 can bind L-selectin and has been implicated in cell-cell adhesion events during tethering, although this was not seen in peripheral blood T cells. ${ }^{32}$ This could be occurring in our assay, especially as we did not see an additive effect by blocking the two receptors, but it is difficult to test directly. $\mathrm{L}$-selectin is not well expressed on lung derived T cells which argues against this receptor being involved in recruitment to the lung. However, it is possible that it is shed on migration into the lung, especially in view of the activated phenotype of lung $\mathrm{T}$ cells. P-selectin gene deleted mice have impaired migration of T cells into the lung in a model of Th2 mediated inflammation, although the anatomical differences between the mouse lung reduce the relevance of this animal as a model for lymphocyte homing. ${ }^{33}$

The lung is comprised of a number of distinct compartments which have different control pathways for leucocyte extravasation. In particular, the bronchial compartment is part of the systemic circulation and therefore requires a tethering step for adhesion to occur, whereas the alveolar bed is supplied by the low pressure pulmonary circulation and migration occurs largely through pulmonary capillaries where adhesion receptors appear to be less important. Consistent with this we found that P-selectin was not expressed on pulmonary capillaries and in preliminary experiments peripheral blood T cells failed to bind to alveolar tissue under the conditions used for this study. This further emphasises the potentially crucial differences in leucocyte trafficking into these two compartments.

In our previous studies we have used inflamed tissue whereas in this study we were attempting to model normal trafficking behaviour, although we accept that resected lung is only an approximation of normal as virtually all patients would have been smokers with lung cancer. Nonetheless, the weak expression of E-selectin suggests that the tissue was not grossly inflamed. It would therefore seem that the adhesion receptors involved in $\mathrm{T}$ cell migration in health and inflammatory airway disease are similar and that the same subsets of peripheral blood $\mathrm{T}$ cells are attracted during inflammation, although presumably in greater numbers as a result of increased expression of chemokines and adhesion receptors. Consistent with this observation, we did not see any difference in our previous study between the chemokine receptor phenotype of $\mathrm{T}$ cells from normal and mildly asthmatic subjects. ${ }^{27}$ $\mathrm{P}$-selectin is stored in intracellular Weibel-Palade bodies and, using the FSA, it is not possible to exclude the possibility that some $\mathrm{T}$ cells are binding to intracellular P-selectin. It was originally thought that P-selectin was only involved in acute inflammatory responses as a result of short term upregulation from these stores. It was also reported that $\mathrm{P}$ and E-selectin were involved in mediating migration of in vitro polarised Thl but not Th2 cells into inflamed tissues.[34 35 $]^{36}$ However, chronic expression of P-selectin can be seen both in vivo and in vitro, especially in the presence of IL-4 and IL-13. ${ }^{28} 3738$ The observation that $\mathrm{P}$-selectin expression is regulated specifically by Th2 cytokines and selectively mediates eosinophil recruitment suggests that it has at least as great a role in Th2 as Thl mediated inflammation. The extent to which P-selectin is involved in Thl versus Th2 recruitment therefore remains unclear.

The major integrin involved in T cell adhesion was LFA- 1 which has been implicated as the major receptor involved in $\mathrm{T}$ cell recruitment in many organs and therefore does not direct organ specific recruitment. Both ICAM-1 and ICAM-2 are well established as ligands for LFA-1. It was perhaps surprising 
that VLA-4/VCAM-1 were not involved but this was consistent with our findings in the nasal polyp endothelium system using a different set of VCAM- 1 antibodies.

In summary, we have characterised the adhesion pathways involved in $\mathrm{T}$ cell adhesion to bronchial epithelium. No specific lung homing receptor has been described, but there appears to be a clear distinction between lung homing cells which appear to use non-CLA expressing PSGL-l binding to bind to P-selectin, gut homing cells which use $\alpha 4 \beta 7$ binding to MAdCAM-1, and skin homing cells which use CLA positive PSGL-1 binding to E-selectin. Further studies are required to determine the molecular specificity of this distinction.

\section{ACKNOWLEDGEMENTS}

This study was funded in part by a travel grant from the Wellcome Trust to Professor Wardlaw. The authors are grateful for the advice and support of Dr Eugene Butcher in whose laboratory this work was initiated.

\section{Authors' affiliations}

M P Ainslie, C A McNulty, T Huynh, F A Symon, A J Wardlaw,

Division of Respiratory Medicine, Institute for Lung Health,

Leicester-Warwick Medical School, Glenfield Hospital, Leicester, UK

\section{REFERENCES}

1 Lipscomb MF, Bice DE, Lyons CR, et al. The regulation of pulmonary immunity. Adv Immunol 1995:59:369-455.

2 Kay AB. Role of T cells in asthma. Chem Immunol 1998;71:178-91.

3 Butcher EC, Williams M, Youngman K, et al. lymphocyte trafficking and regional immunity. Chem Immunol 1999;72:209-53.

4 Mackay CR, Marston WL, Dudler L. Naive and memory T cells show distinct pathways of lymphocyte recirculation. J Exp Med 1990;171:801-17.

5 Butcher EC. Leukocyte-endothelial cell recognition: three (or more) steps to specificity and diversity. Cell 1991;67:1033-6.

6 Symon FA, Wardlaw AJ. Selectins and their counter receptors: a bitter sweet attraction. Thorax 1996:51:1155-7.

7 Kansas GS. Selectins and their ligands: current concepts and controversies. Blood 1996;88:3259-87.

8 Vachino G, Chang X, Veldman GM, et al. P-selectin glycoprotein ligand-1 is the major counter-receptor for P-selectin on stimulated T cells and is widely distributed in non- functional form on many lymphocytic cells. J Biol Chem 1995;270:21966-74.

9 Somers WS, Tang J, Shaw GD, et al. Insights into the molecular basis of leukocyte tethering and rolling revealed by structures of $\mathrm{P}$ - and $\mathrm{E}$-selectin bound to SLe(X) and PSGL-1. Cell 2000;103:467-79.

10 Berlin C, Bargatze RF, Campbell JJ, et al. Alpha 4 integrins mediate lymphocyte attachment and rolling under physiologic flow. Cell 1995;80:413-22.

11 Gonzalez-Amaro R, Sanchez-Madrid F. Cell adhesion molecules: selectins and integrins. Crit Rev Immunol 1999;19:389-429.

12 Briskin M, Winsor-Hines D, Shyjan A, et al. Human mucosal addressin cell adhesion molecule-1 is preferentially expressed in intestinal tract and associated lymphoid tissue. Am J Pathol 1997;151:97-110.

13 Campbell JJ, Bowman EP, Murphy K, et al. 6-C-kine (SLC), a lymphocyte adhesion-triggering chemokine expressed by high endothelium, is an agonist for the MIP-3beta receptor CCR7. J Cell Biol 1998; 141:1053-9.

14 Mackay CR. Chemokines: immunology's high impact factors. Nature Immunol 2001;2:95-101.

15 Campbell JJ, Butcher EC. Chemokines in tissue-specific and microenvironment-specific lymphocyte homing. Curr Opin Immunol 2000;12:336-41.

16 Hemmerich S, Rosen SD. Carbohydrate sulfotransferases in lymphocyte homing. Glycobiology 2000;10:849-56.
17 Rosen SD. Endothelial ligands for L-selectin: from lymphocyte recirculation to allograft rejection. Am J Pathol 1999;155:1013-20.

18 Sallusto $F$, Lenig D, Forster R, et al. Two subsets of memory $T$ lymphocytes with distinct homing potentials and effector functions (see comments). Nature 1999;401:708-12.

19 Zabel BA, Agace WW, Campbell JJ, et al. Human G protein-coupled receptor GPR-9-6/CC chemokine receptor 9 is selectively expressed on intestinal homing T lymphocytes, mucosal lymphocytes, and thymocytes and is required for thymus-expressed chemokine-mediated chemotaxis. J Exp Med 1999;190:1241-56.

20 Kunkel EJ, Campbell JJ, Haraldsen G, et al. Lymphocyte CC chemokine receptor 9 and epithelial thymus-expressed chemokine (TECK) expression distinguish the small intestinal immune compartment: epithelial expression of tissue-specific chemokines as an organizing principle in regional immunity. J Exp Med 2000;192:761-8.

21 Picker LJ, Kishimoto TK, Smith CW, et al. ELAM-1 is an adhesion molecule for skin-homing T cells. Nature 1991;349:796-9.

22 Campbell JJ, Haraldsen G, Pan J, et al. The chemokine receptor CCR4 in vascular recognition by cutaneous but not intestinal memory $T$ cells. Nature 1999;400:776-80.

23 Homey B, Wang W, Soto $\mathrm{H}$, et al. Cutting edge: the orphan chemokine receptor $G$ protein-coupled receptor-2 (GPR-2, CCR 10) binds the skin-associated chemokine CCL27 (CTACK/ALP/ILC). J Immunol 2000; 164:3465-70

24 Csencsits KL, Jutila MA, Pascual DW. Nasal-associated lymphoid tissue: phenotypic and functional evidence for the primary role of peripheral node addressin in naive lymphocyte adhesion to high endothelial venules in a mucosal site. J Immunol 1999;163:1382-9.

25 Abitorabi MA, Mackay CR, Jerome EH, et al. Differential expression of homing molecules on recirculating lymphocytes from sheep gut, peripheral and lung lymph. J Immunol 1996;156:31 11-7.

26 Picker LJ, Martin RJ, Trumble A, et al. Differential expression of lymphocyte homing receptors by human memory/effector T cells in pulmonary versus cutaneous immune effector sites. Eur J Immunol 1994;24:1269-77

27 Campbell JJ, Brightling CE, Symon FA, et al. Expression of chemokine receptors by lung $T$ cells from normal and asthmatic subjects. J Immunol $2001 ; 166: 2842-8$

28 Symon FA, Walsh GM, Watson SR, et al. Eosinophil adhesion to nasal polyp endothelium is P-selectin-dependent. J Exp Med 1994;180:371-6.

29 Symon FA, McNulty CA, Wardlaw AJ. P- and L-selectin mediate binding of T cells to chronically inflamed human airway endothelium. Eur J Immunol 1999:29:1324-33.

30 Bentley AM, Durham SR, Robinson DS, et al. Expression of endothelial and leukocyte adhesion molecules interacellular adhesion molecule-1, E-selectin, and vascular cell adhesion molecule- 1 in the bronchial mucosa in steady-state and allergen-induced asthma. J Allergy Clin Immunol 1993;92:857-68.

31 McNulty CA, Symon FA, Wardlaw AJ. Characterization of the integrin and activation steps mediating human eosinophil and neutrophil adhesion to chronically inflamed airway endothelium. Am J Respir Cell Mol Biol 1999;20:1251-9.

32 Alon R, Fuhlbrigge RC, Finger EB, et al. Interactions through L-selectin between leukocytes and adherent leukocytes nucleate rolling adhesions on selectins and VCAM-1 in shear flow. J Cell Biol 1996;135:849-65.

33 Broide DH, Sullivan S, Gifford T, et al. Inhibition of pulmonary eosinophilia in P-selectin- and ICAM-1-deficient mice. Am J Respir Cell Mol Biol 1998; 18:218-25.

34 Austrup F, Vestweber D, Borges $\mathrm{E}$, et al. P- and E-selectin mediate recruitment of T-helper-1 but not T-helper-2 cells into inflammed tissues. Nature 1997; 385:81-3

35 Borges E, Tietz W, Steegmaier M, et al. P-selectin glycoprotein ligand-1 (PSGL-1) on T helper 1 but not on T helper 2 cells binds to P-selectin and supports migration into inflamed skin. J Exp Med 1997;185:573-8.

36 Xie H, Lim YC, Luscinskas FW, et al. Acquisition of selectin binding and peripheral homing properties by CD4(+) and CD8(+) T cells. J Exp Med $1999 ; 189: 1765-76$

37 Yao L, Pan J, Setiadi $H$, et al. Interleukin 4 or oncostatin $M$ induces a prolonged increase in P-selectin mRNA and protein in human endothelia cells. J Exp Med 1996;184:81-92.

38 Woltmann G, McNulty CA, Dewson G, et al. Interleukin-13 induces PSGL-1/P-selectin-dependent adhesion of eosinophils, but not neutrophils, to human umbilical vein endothelial cells under flow. Blood 2000:95:3146-52. 